\title{
Ultrastructural and Aneuploidy Studies in Sperm from Patients with His- tory of Consanguinity
}

\author{
E. Moretti and G. Collodel ${ }^{*}$ \\ Department of Molecular and Developmental Medicine, University of Siena, Policlinico Le Scotte, Viale Bracci 14, \\ 53100 Siena, Italy
}

\begin{abstract}
The data on the role of consanguinity in male infertility are scarce. The presence of systematic sperm defects in consanguineous respect to non-consanguineous population was demonstrated. Systematic defects are characterized by a specific alteration present in sperm for the whole life of the carrier.

This study was aimed at exploring the sperm quality in selected infertile consanguineous patients (group 3) with nonsystematic sperm defects, compared with that of infertile non-consanguineous patients (group 2) and that of proven fertility subjects (group 1). Sperm analysis was performed by light and transmission electron microscopy (TEM). TEM data was mathematically elaborated. Fluorescence in situ hybridization (FISH) analysis for chromosome $18, \mathrm{X}$ and $\mathrm{Y}$ was performed in sperm nuclei for aneuploidy evaluation. The progressive motility was reduced in groups $2(p<0.05)$ and 3 $(\mathrm{p}<0.01)$, compared to group 1 , and in group 3 versus group $2(\mathrm{p}<0.01)$. Regarding TEM scores, fertility index was reduced in group $3(\mathrm{p}<0.01)$; the percentages of sperm apoptosis, necrosis and immaturity were significantly higher in groups 2 and 3 versus group 1 and in group $3 v s$. group 2 (except for necrosis). FISH analysis showed increased frequency of diploidy ( $\mathrm{p}<0.01)$ in groups 2 and $3 v s$. group 1 .

The consanguinity seems to influence the spermatogenetic process from a morphological point of view, by reducing the motility and fertility index and increasing sperm apoptosis and immaturity; chromosome meiotic segregation was not affected by the condition of consanguinity.
\end{abstract}

Keywords: Consanguinity, ejaculated sperm, FISH, non-systematic sperm defect, systematic sperm defect, TEM.

\section{INTRODUCTION}

Male infertility is a significant problem in humans and it may be caused by different pathologies, such as anatomical problems, infections, hormonal imbalances, chromosomal alterations or gene anomalies, although the cause of infertility remains unknown for $30 \%$ of infertile men [1]. In the last decades particular attention has been directed to the study of genetic of male infertility and a starting point was represented by the observation that human male oligoasthenozoospemia has a familial component [2]. A key indication on the genetic origin of some manifestations of male infertility was provided by a study focused on the analysis of sperm morphology in a large group of infertile patients characterized by different degrees of consanguinity [3].

This research was based on transmission electron microscopy (TEM) observations that allowed the exploration of the "ultrastructure world" and the study of the different organelles rigorously characterizing sperm abnormalities. TEM approach is able to define two main forms of ultrastructural sperm anomalies: non-systematic or non-specific sperm de

*Address correspondence to this author at the Department of Molecular and Developmental Medicine, University of Siena; Policlinico Le Scotte, Viale Bracci 14, 53100 Siena, Italy; Tel: 0577/233504; Fax: 0577/233527;

E-mail: giulia.collodel@unisi.it fects and systematic sperm defects $[3,4]$. The first and the most frequent type includes a heterogeneous combination of alterations randomly affecting the head and the tail organelles in different percentages, generally related to andrological pathologies (i.e. infections and varicocele) or to other endogenous or exogenous factors [4-7]. Systematic sperm defects are rare and are characterized by a very homogeneous microscopic pattern with a peculiar defect present in the vast majority of the sperm population of affected patients. These defects show family clustering and are significantly more common in individuals with a history of consanguinity; the most frequent systematic sperm defects are: "globozoospermia" or round head acrosome-less spermatozoa [8], "defects of head neck attachment" and "acephalic sperm" [4], "dysplasia of fibrous sheath" (DFS) [9] and "primary ciliary dyskinesia" (PCD) [10, 11].

Baccetti et al. [3] demonstrated that such systematic sperm defects were present in $27 \%$ of consanguineous infertile males and $1 \%$ of non-consanguineous infertile patients. The characteristics and the familial incidence of these kinds of sperm defects were then reviewed by Chemes and Rawe [4]; in addition, sperm tail agenesis in patients with second degree cousin parents has been described using TEM [12].

Regarding the sperm quality of consanguineous infertile males without systematic sperm defects, the data is rather 
scarce: to the best of our knowledge, only one paper described that the progressive sperm motility in a group of consanguineous males is significantly reduced respect to sperm motility from a group of non-consanguineous patients [3].

This study was aimed at exploring the sperm quality in a group of selected consanguineous infertile patients, focusing in particular on non-systematic sperm defects.

Sperm analysis was performed by TEM and the results were quantified by a mathematical formula based on probability calculation in force in our laboratory for many years. This method provides a score, as a fertility index represented by the number of sperm free of ultrastructural defects in an ejaculate and the percentage of sperm pathologies such as apoptosis, immaturity and necrosis [13, 14].

In addition, fluorescence in situ hybridization (FISH) analysis for chromosome $18, \mathrm{X}$ and $\mathrm{Y}$ has been performed in sperm nuclei for a direct evaluation of aneuploidies in male gametes.

\section{MATERIALS AND METHODOLOGY}

\section{Patients}

Among 750 male patients addressed at the Interdepartmental Centre for Research and Therapy of Male Infertility, University of Siena, between 2002 and 2013, 27 individuals (aged 30-46 years) declared a history of consanguinity when they were interviewed about their case histories, reproductive problems and family backgrounds. All patients included in this study were born and lived in Italy. The inclusion criteria for this study encompassed: a normal 46, XY karyotype evaluated by conventional cytogenetic analysis, no history of diabetes, radiotherapy, chemotherapy, chronic illness or medications, a hormone ( $\mathrm{FSH}, \mathrm{LH}, \mathrm{T}$ ) profile in the ranges, a BMI $<25 \mathrm{~kg} / \mathrm{m}^{2}$. Patients were non smokers. In addition, microbiological investigations of semen specimens and urethral fluids were performed in order to exclude the presence of genitourinary infections. Finally, clinical and physical examinations and scrotal Eco-color Doppler were carried out in all patients in order to investigate the presence of varicocele or other anatomical problems.

In the group of non-consanguineous infertile patients, ten age-matched non-consanguineous idiopathic infertile patients were selected. All infertile patients were individuals that did not obtain pregnancy after two years of unprotected sexual intercourses and satisfy the same inclusion criteria of the consanguineous group. In addition, a group of 25 men with proven fertility [14] were also included for variables comparisons. Fertile men (aged 22 to 40 years) showed a normal karyotype and they were not affected by anatomical problems and infections. These fertile men had fathered one or more children during past three years.

All patients provided informed written consent before inclusion in this study.

\section{Semen Analysis}

Semen was collected by masturbation after 4 days of sexual abstinence and examined after liquefaction for $30 \mathrm{~min}$ at $37{ }^{\circ} \mathrm{C}$. Volume, $\mathrm{pH}$, sperm concentration and motility were evaluated according to WHO $[15,16]$ guidelines. In patients with a sperm concentration of less than 10 million/ml, PCR analysis on DNA extracted from blood lymphocytes (QIAamp DNA Blood kit, QIAGEN, Milan, Italy) was carried out to exclude the presence of $\mathrm{Y}$ chromosome microdeletions Multiplex PCR was performed using the STS markers from the AZF region of the long arm of the Y chromosome. The STS markers included sY84 and sY86 for $\mathrm{AZFa}, \mathrm{sY} 127$ and sY134 for AZFb, and sY158 and sY254 for AZFc [17]. A normal male, a normal female and no template controls were included as internal controls.

\section{Transmission Electron Microscopy}

For electron microscopy, sperm samples were fixed in cold Karnovsky fixative and maintained at $4{ }^{\circ} \mathrm{C}$ for two hours. Fixed semen was washed in $0.1 \mathrm{~mol} / \mathrm{l}$ cacodylate buffer ( $\mathrm{pH}$ 7.2) for 12 hours, postfixed in $1 \%$ buffered osmium tetroxide for one hour at $4{ }^{\circ} \mathrm{C}$, then dehydrated and embedded in Epon Araldite. Ultra-thin sections were cut with a Supernova ultramicrotome (Reickert Jung, Vienna, Austria), mounted on copper grids, stained with uranyl acetate and lead citrate and then observed and photographed with a Philips EM208 electron microscope (Philips Scientifics, Eindhoven, The Netherlands).

For each patient, three hundred ultra-thin sperm sections were analyzed. Major submicroscopic characteristics were recorded by two trained examiners who were blind to the experiment. TEM data was evaluated using the statistical mathematical formula by Baccetti et al. [13]. This formula considers 16 selected submicroscopic characteristics of sperm organelles able to define sperm function and calculates the number of spermatozoa probably free of structural defects (fertility index, FI) and the percentages of three main phenotypic sperm pathologies: immaturity, necrosis and apoptosis [14], each one characterized by typical alterations of organelles. Reduced acrosomes, round or elliptical nuclei with uncondensed chromatin, and the presence of cytoplasmic droplets were the examined characteristics for immaturity. Marginated chromatin and altered shaped nuclei were considered a main ultrastructural markers of apoptosis, whereas spermatozoa with broken plasma membranes, reacted acrosomes, and disrupted chromatin are affected by necrosis.

\section{Fluorescence in Situ Hybridization}

To evaluate aneuploidy frequency, FISH was performed according to Baccetti et al. [18] in the sperm nuclei of all the patients. A combination of $\alpha$-satellite DNA probes (CEP, Chromosome Enumeration Probes, Vysis, IL, USA) for chromosomes $18, \mathrm{X}$, and $\mathrm{Y}$, directly labeled with different fluorochromes, was used. Observation and scoring were performed using a Leitz Aristoplan Optical Microscope (Leica, Wetzlar, Germany), equipped with a fluorescence apparatus with a triple bandpass filter for aqua, orange and green fluorochromes (Vysis), and a monochrome filter for DAPI (4',6-diamidino-2-phenylindole). Whenever possible, nearly 5,000 sperm nuclei were scored for each sample.

\section{Statistical Analysis}

Statistical analysis was performed using the version 8 SAS system (Sas Institute Inc. Cary, NC 27513, USA). The 
Table 1. Age ranges and means and standard deviations of age, BMI, hormone levels in the 3 considered groups: controls (group 1; \#25), infertile non consanguineous patients (group 2; \#10); infertile consanguineous patients (group 3; \#11).

\begin{tabular}{|c|c|c|c|c|c|c|}
\hline Groups & Age Range & Age & BMI & FSH Level & LH Level & Testosterone Level \\
\hline \hline $1(25)$ & $22-40$ & $29.8 \pm 5.3$ & $23.5 \pm 1.9$ & $5.5 \pm 2.6$ & $3.5 \pm 1.3$ & $5.3 \pm 2.1$ \\
\hline $2(10)$ & $26-36$ & $30.4 \pm 3.4$ & $23.6 \pm 2.2$ & $5.5 \pm 2.8$ & $4.2 \pm 2.1$ & $5.4 \pm 2.3$ \\
\hline $3(11)$ & $24-42$ & $31.6 \pm 4.9$ & $23.6 \pm 2.3$ & $5.3 \pm 3.5$ & $3.0 \pm 2.4$ & $4.6 \pm 1.7$ \\
\hline & & & $<25 \mathrm{~kg} / \mathrm{m}^{2}$ & $0.7-11.0 \mathrm{mU} / \mathrm{ml}$ & $0.8-8.0 \mathrm{U} / \mathrm{L}$ & $2.8-11.4 \mathrm{mg} / \mathrm{ml}$ \\
\hline
\end{tabular}

Table 2. Means and standard deviations of semen variables in the 3 considered groups: controls (group 1; \#25), infertile non consanguineous patients (group 2; \#10); infertile consanguineous patients (group 3; \#11). Sperm concentration and motility were evaluated by light microscopy (LM), the fertility index (FI), apoptosis, necrosis and immaturity by transmission electron microscopy (TEM), diploidies and disomies by fluorescence in situ hybridization (FISH). Statistics were also reported. The significant values reported in the last column were obtained by applying the Mann Whitney U-test among the pairs of groups. * $\mathbf{p}<0.05 ; *$; $<0.01$.

\begin{tabular}{|c|c|c|c|c|c|c|}
\hline & Variables & \multicolumn{3}{|c|}{ Diagnosis } & \multicolumn{2}{|c|}{ Statistics } \\
\hline & & $\# 25$ & \# 10 & \# 11 & & \\
\hline \multirow{4}{*}{ LM/TEM } & Sperm $/ \mathrm{ml} \times 10^{6}$ & $74.26(43.28)$ & $54.83(46.07)$ & $40.24(37.81)$ & 0.0467 & $1 \times 3 *$ \\
\hline & Fertility index $(\mathrm{FI})$ & $\begin{array}{c}7,386,080 \\
(10,464,288)\end{array}$ & $\begin{array}{c}432,234 \\
(503,689)\end{array}$ & $\begin{array}{c}619,917 \\
(1,104,566)\end{array}$ & $<0.0001$ & $1 \times 2^{* *} ; 1 \times 3^{* *} ; 2 \times 3^{* *}$ \\
\hline & Apoptosis & $4.05(2.04)$ & $6.89(3.84)$ & $9.89(6.05)$ & 0.013 & $1 \times 2 * ; 1 \times 3^{* *} ; 2 \times 3^{*}$ \\
\hline & Necrosis & $32.12(10.57)$ & 46.09 (11.64) & $40.34(14.84)$ & 0.0133 & $1 \times 2^{* *} ; 1 \times 3^{* *}$ \\
\hline \multirow[t]{2}{*}{ FISH } & 18 disomy \% & $0.103(0.052)$ & $0.102(0.017)$ & $0.116(0.042)$ & 0.527 & \\
\hline & $\begin{array}{c}\text { Sex chromosomes } \\
\text { disomy } \%\end{array}$ & $0.242(0.109)$ & $0.298(0.159)$ & $0.428(0.344)$ & 0.1562 & \\
\hline
\end{tabular}

comparisons between the considered variables related to the groups of consanguineous patients, infertile patients and controls were performed by Kruskal-Wallis test; when a statistically significant difference was found among the groups, the Mann-Whitney U-test was then used between pairs of groups. $\mathrm{P}<0.05$ was considered significant.

\section{RESULTS}

In the studied period, the semen analysis of 750 male patients was carried out in our laboratory. Twenty seven males (3.6\%) were consanguineous. Among 27 individuals with a history of consanguinity we selected a group of 11 patients with idiopathic infertility. Six patients were excluded for the presence of varicocele and/or genitourinary infection and/or hormonal imbalance, one patient was azoospermic, one man was carrier of a translocation [46, XY t(13;14)] and 8 patients $(29.6 \%)$ showed a systematic sperm defect (4 men showed sperm with DFS, 1 man had round headed sperm, 1 patient showed Kartagener syndrome, 2 other subjects displayed composed defects of supposed genetic origin [19, 20]. Selected patients showed different degrees of consanguinity (the parents of six patients were first degree cousins; parents of one patient were second degree cousins; grand-parents of two subjects were first degree cousins; two infertile patients had brothers with systematic sperm defects). In the group of non-consanguineous patients (723 individuals) 7 patients (around 1\%) showed a systematic sperm defect.

The age range, the mean \pm standard deviation of age, BMI and hormone levels in the studied groups were reported (Table 1). The semen quality of selected group of consanguineous infertile patients (group 3) was compared with those of a group of non-consanguineous infertile patients (group 2) and with a group of subjects with proven fertility (group 1); the results of the comparison are reported in Table 2. 
Sperm concentration was normal $[15,16]$ in all analysed groups even if significantly reduced in group 3 respect to group 1 ( $\mathrm{p}<0.05$ ) (Table 2 ). The progressive motility was significantly reduced in both groups of infertile patients compared to the values of fertile individuals (group $1 \mathrm{vs}$. group 2, $\mathrm{p}<0.05$; group 1 vs. group $3, \mathrm{p}<0.01$ ); in addition, sperm motility of consanguineous patients was significantly decreased $(\mathrm{p}<0.01)$ respect to that of non-consanguineous infertile males.

Regarding TEM scores, FI was significantly reduced in group 3 ( $p<0.01$ ) compared to that observed in group 1 and group 2 and in group 2 vs. group $1(\mathrm{p}<0.01)$ (Table 2$)$.

The percentages of sperm apoptosis, necrosis and immaturity were, as expected, significantly higher in both groups of infertile patients respect to controls (Table 2); in addition, the sperm pathologies were significantly more frequent in consanguineous infertile patients then in sperm from nonconsanguineous infertile patients, excepted for necrosis.

FISH analysis was performed in sperm nuclei of 11 consanguineous infertile patients (group 3) and 10 nonconsanguineous infertile patients (group 2). A total number of 193,062 sperm nuclei was scored in group 3 and a total number of 171,547 in group 2. The mean frequencies of aneuploidy of chromosomes 18, X, Y are summarised in Table 2. The frequency of diploidy was significantly higher $(\mathrm{p}<0.01)$ in groups 2 and 3 than in controls (group 1).

\section{DISCUSSION}

Consanguineous marriages are practiced in about one tenth of the world's population, and are thus more widespread than commonly perceived, although their overall distribution is not uniform [21]. In countries where inbreeding is common, a concomitant increase in the frequency of autosomal recessive disorders is generally observed by virtue of the biparental inheritance of the same ancestral haplotype on whom the recessive mutations reside. However, some novel aspects of consanguineous marriages are recently reported. In particular, Denic et al. [22] proposed that consanguineous marriages increase the speed of selection of beneficial recessive and co-dominant alleles, enhancing the fitness of a population under specific ecological conditions; for example, in countries where malaria is endemic, the prevalence of inbreeding and the frequency of alleles protective against malaria are both high.

Concerning the role of consanguinity in male infertility, the current data are quite scarce. The question that seems clearly established concerns the increased presence of systematic sperm defects in consanguineous respect to non consanguineous population [3, 4, 20, 23]; systematic defects are quite rare and are characterized by an identical, specific alteration of sperm cells. The patients carrier of systematic defects are sterile because these alterations are untreatable and are present in the ejaculates for their entire life. The results obtained in this study, pertaining to systematic sperm defects, confirm the data reported in the literature.

In the present research, we particularly focused on the sperm quality of a group of selected patients with various degrees of consanguinity who did not show the systematic sperm defects, but a heterogeneous combination of altera- tions randomly affecting in different percentage the sperm head and the tail organelles; the considered variables were compared with those of a group of idiopathic patients free of biological relationship and with those of a group of proven fertility individuals. As expected, the sperm quality of fertile individuals was enhanced compared to that of both the infertile groups. The percentage of sperm motility was significantly lower in the group of consanguineous patients compared to that evaluated in group of non-consanguineous infertile subjects, as also reported by Baccetti et al. [3]. The data concerning the scores provided by TEM analysis mathematically elaborated are novel. TEM scores were, as expected, decreased in both groups of infertile men, respect to those observed in the control. The fertility index was reduced and the percentages of sperm pathologies, namely apoptosis and immaturity were significantly increased in sperm of consanguineous infertile patients compared to those of non-consanguineous infertile patients.

The consanguinity in the familial history of analyzed patients led us to suppose that genetic alterations could be responsible of the reduced semen quality in the group of infertile consanguineous patients in respect to that of infertile non consanguineous patients.

The sperm analysis of the considered groups was integrated with FISH analysis that is a useful technique for estimating aneuploidy frequencies directly in interphase cells, such as spermatozoa [24]. The option to apply FISH analysis to the sperm of these individuals originated from the observation that this technology, coupled with TEM analysis, provided a satisfying characterization of sperm anomalies [2527] from a morphological and meiotic point of view. Elevated FISH values in sperm were reported in men with altered sperm ultrastructure [28-30]. In this particular study, we found an increase in the frequency of sperm diploidy and sex disomy in the groups of infertile consanguineous and non-consanguineous patients compared to that found in fertile men, but only the percentage of diploidy was significantly increased in both groups of infertile patients. Diploidy of sperm cells could be generated by binucleate sperm head or by diploid nuclei with the presence of double sets of 18 , $\mathrm{X}, \mathrm{Y}$ chromosomes [31]. Since ejaculated sperm in both infertile groups was morphologically compromised and showed high percentage of sperm immaturity, it is plausible that the increased diploidy could be due to sperm with double nuclei. A similar meiotic behavior in the consanguineous and non-consanguineous infertile patients may suggest that consanguinity does not seem to impair meiotic segregation of chromosomes.

\section{CONCLUSION}

Consanguinity may adversely affect the spermatogenetic process, in particular by causing a reduction of motility and FI, which is concomitant with an increased sperm apoptosis and immaturity. We are aware that the patient group is small and further studies on a larger sample will help to better understand the role of consanguinity in male infertility; however, it should be considered that our patients were enrolled after following stringent selection criteria to exclude the presence of other pathologies involved in male infertility. 


\section{CONFLICT OF INTEREST}

The authors confirm that this article content has no conflict of interest.

\section{ACKNOWLEDGEMENTS}

Declared none.

\section{REFERENCES}

[1] Cavallini G. Male idiopathic oligoasthenoteratozoospermia. Asian J Androl 2006; 8: 143-57.

[2] Lilford R, Jones AM, Bishop DT, Thornton J, Mueller R. Casecontrol study of whether subfertility in men is familial. BMJ 1994; 309: 570-3.

[3] Baccetti B, Capitani S, Collodel G, et al. Genetic sperm defects and consanguinity. Hum Reprod 2001; 16: 1365-71.

[4] Chemes HE, Rawe VY. Sperm pathology: a step beyond descriptive morphology. Origin, characterization and fertility potential of abnormal sperm phenotypes in infertile men. Hum Reprod Update 2003; 9: 405-28.

[5] Jurewicz J, Hanke W, Radwan M, Bonde JP. Environmental factors and semen quality. Int J Occup Med Environ Health 2009; 22: 30529

[6] Collodel G, Capitani S, Pammolli A, Giannerini V, Geminiani M, Moretti E. Semen quality of male idiopathic infertile smokers and nonsmokers: an ultrastructural study. J Androl 2010; 31: 108-13.

[7] Fariello RM, Pariz JR, Spaine DM, Cedenho AP, Bertolla RP, Fraietta R. Association between obesity and alteration of sperm DNA integrity and mitochondrial activity. BJU Int 2012; 110: 8637.

[8] Schirren CG, Holstein AF, Schirren C. Uber die morphogenese rund-kopfiger spermatozoen des menschen. Andrologia 1971; 3: 117-25.

[9] Chemes HE, Brugo S, Zanchetti F, Carrere C, Lavieri JC. Dysplasia of the fibrous sheath: an ultrastructural defect of human spermatozoa associated with sperm immotility and primary sterility. Fertil Steril 1987; 48: 664-9.

[10] Afzelius BA. A human syndrome caused by immotile cilia. Science 1976, 193: 317-9.

[11] Escalier D, David G. Pathology of the cytoskeleton of the human sperm flagellum: axonemal and peri-axonemal anomalies. Biol Cell 1984; 50: 37-52.

[12] Latini M, Gandini L, Lenzi A, Romanelli F. Sperm tail agenesis in a case of consanguinity. Fertil Steril 2004; 81: 1688-91.

[13] Baccetti B, Bernieri G, Burrini AG, et al. Notulae seminologicae. 5. Mathematical evaluation of interdependent submicroscopic sperm alterations. J Androl 1995; 16: 356-71.

[14] Collodel G, Moretti E. Morphology and meiotic segregation in spermatozoa from men of proven fertility. J Androl 2008; 29: 106-
[15] World Health Organization. WHO Laboratory Manual for the Examination of Human Semen and Sperm-Cervical Mucus Interaction. Cambridge: Cambridge University Press 1999.

[16] World Health Organization. WHO laboratory manual for the examination and processing of human semen. $5^{\text {th }}$ ed, WHO Press, Geneva: Switzerland 2010

[17] Simoni M, Bakker E, Krausz C. EAA/EMQN best practice guidelines for molecular diagnosis of Y-chromosomal microdeletions: state of the art 2004. Int J Androl 2004; 27: 240-9.

[18] Baccetti B, Bruni E, Collodel G, et al. 10, 15 reciprocal translocation in an infertile man: ultrastructural and fluorescence in-situ hybridization sperm study: case report. Hum Reprod 2003; 18: 23028.

[19] Baccetti B, Bruni E, Gambera L, Moretti E, Piomboni P. An ultrastructural and immunocytochemical study of a rare genetic sperm tail defect that causes infertility in humans. Fertil Steril 2004; 82: 463-8.

[20] Collodel G, Federico MG, Pascarelli NA, Geminiani M, Renieri T, Moretti E. A case of severe asthenozoospermia: a novel sperm tail defect of possible genetic origin identified by electron microscopy and immunocytochemistry. Fertil Steril 2011; 95: 289.e11-6.

[21] Alkuraya FS. Impact of new genomic tools on the practice of clinical genetics in consanguineous populations: the Saudi experience. Clin Genet 2013; 84: 203-8.

[22] Denic S, Nagelkerke N, Agarwal MM. On some novel aspects of consanguineous marriages. Public Health Genomics 2011; 14: 1628.

[23] Escalier D, Albert M. New fibrous sheath anomaly in spermatozoa of men with consanguinity. Fertil Steril 2006; 86: 219.e1-9.

[24] Martin RH. Cytogenetic determinants of male infertility. Hum Reprod Update 2008; 14: 379-90.

[25] Perdrix A, Travers A, Chelli MH, et al. Assessment of acrosome and nuclear abnormalities in human spermatozoa with large vacuoles. Hum Reprod 2011; 26: 47-58.

[26] Collodel G, Giannerini V, Pascarelli AN, Federico MG, Comodo F, Moretti E. TEM and FISH studies in sperm from men of couples with recurrent pregnancy loss. Andrologia 2009; 41: 352-60.

[27] Prisant N, Escalier D, Soufir JC, et al. Ultrastructural nuclear defects and increased chromosome aneuploidies in spermatozoa with elongated heads. Hum Reprod 2007; 22: 1052-9.

[28] Collodel G, Moretti E. Sperm morphology and aneuploidies: defects of supposed genetic origin. Andrologia 2006; 38: 208-15.

[29] Baccetti B, Bruni E, Collodel G, Mancini S, Piomboni P, Moretti E. Studies on varicocele III. Ultrastructural studies of spermatozoa from infertile males with varicocele and $18, \mathrm{X}$ and $\mathrm{Y}$ aneuploidies. J Androl 2006; 27: 94-101.

[30] Moretti E, Di Cairano G, Capitani S, Scapigliati G, Baccetti B, Collodel G. Cryptorchidism and semen quality: a TEM and molecular study. J Androl 2007; 28: 194-9.

[31] Baccetti B, Collodel G, Marzella R, et al. Ultrastructural studies of spermatozoa from infertile males with Robertsonian translocations and 18, X, Y aneuploidies. Hum Reprod 2005; 20: 2295-300.

Received: March 14, 2014

Revised: June 10, 2014

Accepted: June 10, 2014

(C) Moretti and Collodel; Licensee Bentham Open.

This is an open access article licensed under the terms of the Creative Commons Attribution Non-Commercial License (http://creativecommons.org/licenses/by-nc/3.0/) which permits unrestricted, non-commercial use, distribution and reproduction in any medium, provided the work is properly cited. 